\title{
Stromal cell-derived factor and granulocyte-monocyte colony-stimulating factor form a combined neovasculogenic therapy for ischemic cardiomyopathy
}

\author{
Y. Joseph Woo, MD, ${ }^{a}$ Todd J. Grand, BS, ${ }^{a}$ Mark F. Berry, MD, ${ }^{a}$ Pavan Atluri, MD, ${ }^{\text {a }}$ Mireille A. Moise, MD, ${ }^{a}$ \\ Vivian M. Hsu, BA, ${ }^{a}$ Jeffrey Cohen, ${ }^{a}$ Omar Fisher, BSE, ${ }^{a}$ Jeffrey Burdick, BS, ${ }^{a}$ Matthew Taylor, BS, ${ }^{a}$

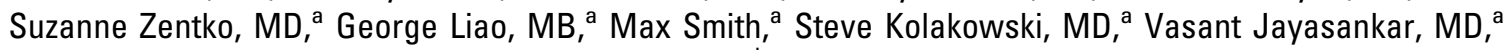 \\ Timothy J. Gardner, MD, a and H. Lee Sweeney, $\mathrm{PhD}^{\mathrm{b}}$
}

From the Division of Cardiothoracic Surgery, Department of Surgery, ${ }^{a}$ and the Department of Physiology, ${ }^{\mathrm{b}}$ University of Pennsylvania School of Medicine, Philadelphia, Pa.

Supported in part by National Institutes of Health (NIH) National Heart, Lung, and Blood Institute/Thoracic Surgery Foundation for Research and Education grant HL072812 (Dr Woo), American Heart Association grant 0465519U (Dr Woo), and NIH Training Grant in Cardiovascular Biology and Medicine T32 HL07843 (Dr Berry and Dr Atluri).

Received for publication Aug 26, 2004; revisions received Oct 12, 2004; accepted for publication Nov 11, 2004

Address for reprints: Y. Joseph Woo, MD, Division of Cardiothoracic Surgery, Department of Surgery, University of Pennsylvania, Silverstein 4, 3400 Spruce St, Philadelphia PA 19104 (E-mail: wooy@uphs.upenn.edu).

J Thorac Cardiovasc Surg 2005;130:321-9

$0022-5223 / \$ 30.00$

Copyright (C) 2005 by The American Association for Thoracic Surgery

doi:10.1016/j.jtcvs.2004.11.041
Objective: Ischemic heart failure is an increasingly prevalent global health concern with major morbidity and mortality. Currently, therapies are limited, and novel revascularization methods might have a role. This study examined enhancing endogenous myocardial revascularization by expanding bone marrow-derived endothelial progenitor cells with the marrow stimulant granulocyte-monocyte colonystimulating factor and recruiting the endothelial progenitor cells with intramyocardial administration of the potent endothelial progenitor cell chemokine stromal cell-derived factor.

Methods: Ischemic cardiomyopathy was induced in Lewis rats $(n=40)$ through left anterior descending coronary artery ligation. After 3 weeks, animals were randomized into 4 groups: saline control, granulocyte-monocyte colony-stimulating factor only (GM-CSF only), stromal cell-derived factor only (SDF only), and combined stromal cell-derived factor/granulocyte-monocyte colony-stimulating factor (SDF/ GM-CSF) $(\mathrm{n}=10$ each). After another 3 weeks, hearts were analyzed for endothelial progenitor cell density by endothelial progenitor cell marker colocalization immunohistochemistry, vasculogenesis by von Willebrand immunohistochemistry, ventricular geometry by hematoxylin-and-eosin microscopy, and in vivo myocardial function with an intracavitary pressure-volume conductance microcatheter.

Results: The saline control, GM-CSF only, and SDF only groups were equivalent. Compared with the saline control group, animals in the SDF/GM-CSF group exhibited increased endothelial progenitor cell density $(21.7 \pm 3.2$ vs $9.6 \pm 3.1$ $\mathrm{CD} 34^{+} /$vascular endothelial growth factor receptor 2-positive cells per high-power field, $P=.01)$. There was enhanced vascularity $(44.1 \pm 5.5$ versus $23.8 \pm 2.2$ von Willebrand factor-positive vessels per high-power field, $P=.007)$. SDF/GM-CSF group animals experienced less adverse ventricular remodeling, as manifested by less cavitary dilatation $(9.8 \pm 0.1 \mathrm{~mm}$ vs $10.1 \pm 0.1 \mathrm{~mm}$ [control], $P=.04)$ and increased border-zone wall thickness $(1.78 \pm 0.19$ vs $1.41 \pm 0.16 \mathrm{~mm}$ [control], $P=.03$ ). (SDF/GM-CSF group animals had improved cardiac function compared with animals in the saline control group (maximum pressure: $93.9 \pm 3.2$ vs $71.7 \pm$ $3.1 \mathrm{~mm} \mathrm{Hg}, P<.001$; maximum dP/dt: $3513 \pm 303$ vs $2602 \pm 201 \mathrm{~mm} \mathrm{Hg} / \mathrm{s}$, $P<.05$; cardiac output: $21.3 \pm 2.7$ vs $13.3 \pm 1.3 \mathrm{~mL} / \mathrm{min}, P<.01$; end-systolic pressure-volume relationship slope: $1.7 \pm 0.4$ vs $0.5 \pm 0.2 \mathrm{~mm} \mathrm{Hg} / \mu \mathrm{L}, P<.01$.)

Conclusion: This novel revascularization strategy of bone marrow stimulation and intramyocardial delivery of the endothelial progenitor cell chemokine stromal cell-derived factor yielded significantly enhanced myocardial endothelial progenitor cell density, vasculogenesis, geometric preservation, and contractility in a model of ischemic cardiomyopathy. 


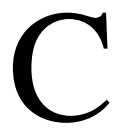
ongestive heart failure (CHF), predominantly a consequence of ischemic heart disease, is an increasingly prevalent global health concern. In the United States there are 5 million patients with $\mathrm{CHF}$ and an estimated additional 5 million persons with asymptomatic left ventricular dysfunction. ${ }^{1} \mathrm{CHF}$ accounts for 1 million hospital admissions and 15 million office visits annually. Current medical therapy for ischemic cardiomyopathy is limited to adjustment of preload and afterload and, in limited circumstances, contractility. Surgical therapies include valvular reconstruction, ventricular assist devices, and transplantation for limited patients. Myocardial revascularization as a therapy for ischemic ventricular dysfunction has produced encouraging improvement in global ventricular function. ${ }^{2}$ These clinical findings have prompted extensive investigation into alternative revascularization strategies because the vast majority of patients with ischemic cardiomyopathy do not have anatomically correctable coronary artery disease. Recent studies have focused on the use of angiogenic factors, such as fibroblast growth factor, ${ }^{3}$ vascular endothelial growth factor (VEGF), ${ }^{4}$ and hepatocyte growth factor. ${ }^{5}$ The latest investigations have focused on the transplantation of ex vivo expanded endothelial progenitor cells (EPCs) as an alternate means of revascularizing ischemic cardiomyopathic hearts. ${ }^{6}$

An EPC is a hematopoietic bone marrow-derived cell that expresses CD34 and VEGF receptor 2 (VEGFR2) and differentiates into an endothelial cell. ${ }^{7}$ EPCs modulate the re-endothelialization of injured blood vessels ${ }^{8}$ and the de novo endothelialization of intravascular implants, such as valvular prostheses, vascular grafts, and ventricular assist devices. ${ }^{9}$ EPCs also participate in neovascularization in regions of ischemia. ${ }^{10}$ Transplantation of EPCs as a practical therapeutic modality is limited by requisite large volumes of source blood or marrow, extensive cell sorting and purification techniques, and lengthy propagation periods.

As an alternative to EPC transplantation, we investigated a strategy of enhancing native EPC recruitment to treat ischemic cardiomyopathy by combining marrow proliferation and localized chemotactic stimuli. Granulocyte-monocyte colony-stimulating factor (GM-CSF), a global bone marrow stimulant, increases total marrow and circulating levels of EPCs. ${ }^{11}$ It is reported that EPC levels begin to increase immediately after GM-CSF administration, continue to increase during repeated administration, and then peak and remain increased for at least 1 week after ceasing administration. ${ }^{11}$ Stromal cell-derived factor $1 \alpha$ (SDF) is an EPC chemokine that promotes the transendothelial migration of EPCs and has been studied primarily for its essential role in embryologic hematopoiesis, vasculogenesis, and cardiogenesis. ${ }^{12}$ SDF is highly conserved across species, with human and rat forms differing in only 2 amino acids. This chemokine interacts with the CXCR-4 receptor, which is highly expressed on EPCs, and induces transient increases in intracellular calcium and catalyzes intracellular actin polymerization required for cell motility. ${ }^{13,14}$ In vitro, SDF increases the length of endothelial cell-lined tubules, and in vivo it produces a dose-dependent increase in tissue endothelial cell density. ${ }^{15}$ After experimental myocardial infarction in rats, myocardial SDF expression is upregulated, thus implicating the initiation of a native EPC-based myocardial reparative mechanism. ${ }^{16}$

SDF has been administered in an ischemic rat hind-limb model as a chemokine for transplanted EPCs, which were expanded in vitro, resulting in increased concentrations of these EPCs within the ischemic tissue and augmented tissue perfusion. ${ }^{17}$ However, when SDF was administered to an ischemic hind limb without the benefit of an expanded circulating pool of transplanted EPCs, the extent of revascularization was insufficient, and autoamputation occurred. Therefore, we postulated that myocardial SDF therapy alone would unlikely be effective, and instead we have proposed an expansion of the native EPC pool through bone marrow stimulation. In this study we devised a strategy of endogenous myocardial repair for ischemic cardiomyopathy through GM-CSF-mediated bone marrow stimulation and SDF-mediated targeted EPC chemokinesis and evaluated the effects on neovasculogenesis, ventricular remodeling, and myocardial function.

\section{Methods}

\section{Animal Care and Biosafety}

Male Lewis rats weighing 250 to $300 \mathrm{~g}$ were obtained from Charles River (Boston, Mass). Food and water were provided ad libitum. This study was performed in accordance with the standard humane care guidelines of the "Guide for the Care and Use of Laboratory Animals" and the Institutional Animal Care and Use Committee of the University of Pennsylvania, which conform to federal guidelines.

\section{Ischemic Cardiomyopathy Model}

An established and highly reproducible rodent model of ischemic cardiomyopathy was used. ${ }^{18}$ This model of postinfarction heart failure also yields a peri-infarct border zone potentially amenable to experimental therapy. Forty rats were anesthetized with ketamine $(75 \mathrm{mg} / \mathrm{kg})$ and xylazine $(7.5 \mathrm{mg} / \mathrm{kg})$, endotracheally intubated with a 14-gauge angiocatheter, and mechanically ventilated (Hallowell EMC, Pittsfield, Mass) with $1 \%$ isoflurane maintenance anesthesia. A thoracotomy was performed in the left fourth intercostal space, and the heart was exposed. A 7-0 polypropylene suture was placed around the mid-left anterior descending coronary artery (LAD), briefly snared to visually verify the territory of myocardial ischemia, and then ligated to produce a large anterolateral myocardial infarction of $30 \%$ of the left ventricle. The extent of infarction was highly reproducible in our own studies and has been documented in the literature as well. ${ }^{5,18}$ The thoracotomy was closed in multiple layers over a temporary thoracostomy catheter. Animals were implanted with identification microchips (BioMedic Data Systems Inc, Seaford, Del) and recovered. 
Three weeks after coronary ligation, while heart failure was developing in the animals, the animals were randomized to 1 of 4 groups in preparation for planned reoperation $(n=10$ for each group): saline control, GM-CSF only, SDF only, and SDF/GMCSF combined therapy. Saline control animals received $200 \mu \mathrm{L}$ of subcutaneous saline on preoperative day 1, intraoperatively, and on postoperative day 1 and a total of $250 \mu \mathrm{L}$ of saline through direct intramyocardial injection with a 30 -gauge needle into 5 sites in the border zone surrounding the prior infarction. GM-CSFtreated animals received doses of $40 \mu \mathrm{g} / \mathrm{kg}$ liquid sargramostim (GM-CSF) in saline (200 $\mu \mathrm{L}$ total) on preoperative day 1 , intraoperatively, and on postoperative day 1 and $250 \mu \mathrm{L}$ of intramyocardial saline. SDF-treated animals received $200 \mu \mathrm{L}$ of subcutaneous saline on preoperative day 1 , intraoperatively, and on postoperative day 1 and intramyocardial injection of $3 \mu \mathrm{g} / \mathrm{kg}$ SDF-1 (BD Biosciences, San Diego, Calif) in $250 \mu \mathrm{L}$ of saline into the infarction border zone. SDF/GM-CSF-treated animals received subcutaneous GM-CSF and intramyocardial SDF injections as described above. The animals were then closed and recovered for another 3 weeks.

\section{Infarct Size Analysis}

At 6 weeks after coronary ligation, after myocardial functional analysis as described below, the hearts were explanted. The left ventricle was filled with OCT compound (Sakura Finetek, Torrance, Calif) through the aorta, and the heart was submerged in an OCT-filled conical tube, frozen in liquid nitrogen, and stored at $-80^{\circ} \mathrm{C}$. By means of a cryostat, multiple slides of $10-\mu \mathrm{m}$ sections from midway between the apex and the point of ligation were prepared for immunohistochemical staining and infarct size analysis. Infarct size was assessed with digital planimetry software (OpenLab, Lexington, Mass) of hematoxylin and eosin-stained sections and compared among groups to exclude any potential differences in myocardial infarct size among groups as possible contributors to observed differences in hemodynamics. Furthermore, a separate group of 10 additional animals were killed 3 weeks after initial LAD ligation to confirm that there was no modulation in infarct size during this second 3-week treatment period, and left ventricular infarct size was determined by using triphenyltetrazolium chloride (1\%) staining (Sigma Chemicals, St Louis, Mo).

\section{EPC Identification}

Frozen sections were washed with cold phosphate-buffered saline (PBS) at room temperature for 10 minutes, fixed with acetone at $-20^{\circ} \mathrm{C}$ for 5 minutes, blocked with $1 \%$ bovine serum albumin in PBS for 30 minutes, and costained with antibodies against CD34 and VEGFR2. Sections were incubated in rabbit anti-mouse CD34 (Santa Cruz Biotech, Santa Cruz, Calif) and goat anti-mouse VEGFR2 (R\&D Systems, Minneapolis, Minn) for 1 hour at room temperature. Sections were washed 3 times in PBS and incubated at room temperature in the dark with 1:40 fluorescein isothiocyanate-conjugated swine anti-goat immunoglobulin (DakoCytomation, Carpinteria, Calif) and 1:400 rhodamine-conjugated donkey anti-rabbit immunoglobulin (Abcam, Cambridge, Mass) secondary antibodies. Slides were washed 3 times with PBS and mounted with Vectashield with 4',6-diamidino-2-phenylindole for nonspecific staining of nuclei (Vector Labs, Burlingame, Calif). Quanti- tative analysis of colocalized antibody expression was conducted with $40 \times$ fluorescent microscopy. Counts were conducted in a group-blinded fashion in 4 fields per specimen along the border zone and averaged for each heart.

\section{Neovascularization Assessment}

Frozen sections were incubated for 10 minutes at room temperature with rabbit anti-human von Willebrand factor (vWF; DakoCytomation) diluted 1:300 in 1\% bovine serum albumin. Slides were then washed 3 times with PBS for 10 minutes and then incubated in the dark at room temperature for 30 minutes with 1:40 fluorescein isothiocyanate-conjugated swine anti-rabbit immunoglobulin (DakoCytomation). Slides were again washed and mounted with Vectashield containing 4',6-diamidino-2-phenylindole vWF-positive blood vessels were quantified by means of $40 \times$ fluorescent microscopy in a group-blinded manner in 4 fields per specimen along the border zone and averaged for each heart.

\section{Ventricular Geometry Analysis}

For chamber size, left ventricular cavitary diameter in both the anteroposterior and mediolateral axes was measured on each section and averaged. For border-zone wall thickness, on each section, 2 measurements were obtained in the immediate peri-infarct region, one on each side of the infarct, medial and lateral, just beyond the zone of fibrosis, and averaged. Measurements were obtained in a group-blinded manner and reported as millimeters \pm SEM.

\section{Hemodynamic Assessment}

Six weeks after initial coronary ligation, when animals are typically in overt heart failure, the rats underwent median sternotomy for myocardial contractility analysis, as previously described. ${ }^{19}$ In brief, a $2 \mathrm{~F}$ pressure-volume conductance catheter (Millar Instruments, Inc, Houston, Tex) was inserted into the left ventricle through the apex of the heart. Multiple cardiac functional parameters were measured, recorded, and analyzed with Chart v4.1.2 software (AD Instruments, Colorado Springs, Colo) and ARIA 1 Pressure Volume Analysis software (Millar Instruments). In addition to steady-state hemodynamic parameters, contractility was determined from pressure-volume relationships obtained by reducing preload through occlusion of the inferior vena cava. Volume measurements were calibrated by 2-point linear interpolation with fixed-volume cuvettes of heparinized rat blood, and parallel conductance was excluded with the hypertonic saline injection technique.

\section{Statistical Methods}

Statistical analyses were performed by 1-way analysis of variance and the Tukey-Kramer Honestly Significant Difference method to test for differences among means. All results were expressed as means \pm SEM.

\section{Results \\ Infarct Size}

At 3 weeks after LAD ligation, rats manifested a mean infarct size of $29.3 \% \pm 2.8 \%(\mathrm{n}=10)$. Six weeks after LAD ligation (3 weeks after assignment to treatment group), 


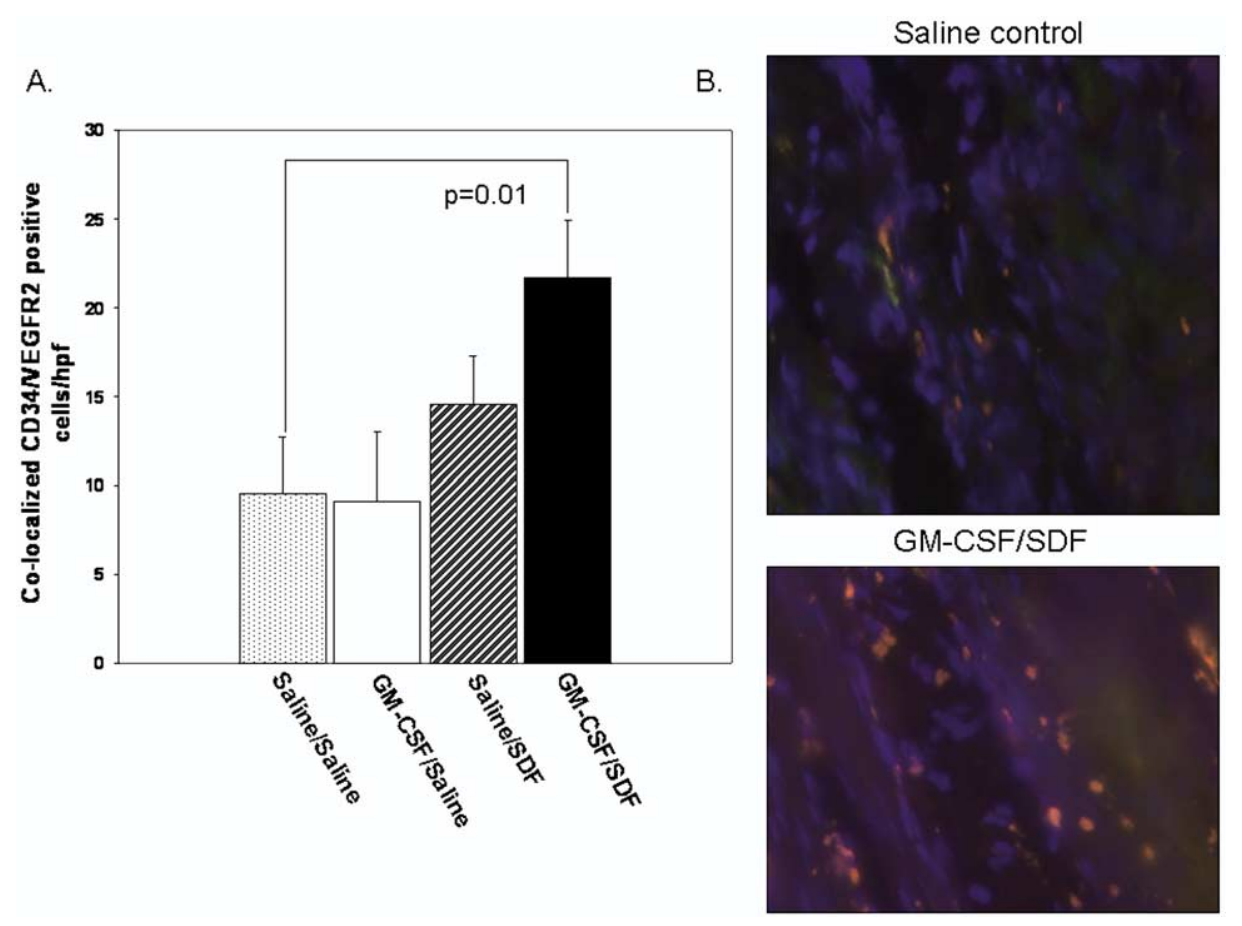

Figure 1. A, Graph of the mean number of CD34 ${ }^{+}$NEGFR2 ${ }^{+}$coexpressing cells per high-power field in hearts from the 4 groups. B, Representative immunostains of saline control and SDF/GM-CSF-treated hearts for CD34 (green), VEGFR2 (red; merged = orange), and DAPI nuclei (blue).

infarct sizes were statistically equivalent among all 4 groups (saline control, $29.1 \% \pm 2.3 \%$; GM-CSF only, $31.6 \% \pm$ $1.8 \%$; SDF only, $30.9 \% \pm 2.9 \%$; and SDF/GM-CSF combined, $29.6 \% \pm 2.4 \% ; P=\mathrm{NS}$ ), thereby validating model reproducibility, consistency among the 4 groups, and absence of infarct modulation.

\section{EPC Identification}

Quantitative analysis of immunohistochemical staining for colocalized EPC markers revealed CD34 and VEGFR2 expression in border-zone regions of SDF/GM-CSF-treated hearts to a far greater extent than in the other 3 groups (Figure 1, A). This consistently colocalized expression of CD34 and VEGFR2 likely indicates regions of immature endothelial cell proliferation. Representative superimposed fluorescent photomicrographs of CD $34^{+} / \mathrm{VEGFR}^{+}$cells in saline control and SDF/GM-CSF-treated hearts are depicted in Figure 1, $B$.

\section{Neovascularization}

Microscopic analysis of immunofluorescent expression for vWF along border-zone regions revealed a significant increase in blood vessel density in SDF/GM-CSF-treated animals (44.1 \pm 5.5 vessels/high-power field [hpf]) com- pared with animals in the saline control group $(23.8 \pm 2.2$ vessels/hpf, $P=.007$; Figure 2, $A$ ). Border-zone myocardium in the GM-CSF-only and SDF-only groups exhibited relatively small and statistically insignificant blood vessel density increases compared with those in the saline control group (GM-CSF only, $24.2 \pm 3.6$ vessels/hpf; SDF only, $31.8 \pm 2.2$ vessels/hpf). Representative vWF immunostains of a saline control heart and an SDF/GM-CSF-treated heart are shown in Figure 2, $B$.

\section{Ventricular Geometry}

Representative hematoxylin and eosin-stained sections of SDF/GM-CSF-treated hearts and control hearts are shown in Figure 3, A. Quantitative analysis of cavitary dilatation and wall thinning in all hearts of all 4 groups revealed that SDF/GM-CSF-treated animals displayed significantly less ventricular dilation than the other 3 groups (saline control, $10.1 \pm 0.1 \mathrm{~mm}$; GM-CSF only, $10.22 \pm 0.1 \mathrm{~mm}$; SDF only, $9.91 \pm 0.01 \mathrm{~mm} ; \mathrm{SDF} / \mathrm{GM}-\mathrm{CSF}, 9.8 \pm 0.12 \mathrm{~mm}$; Figure 3, $B)$. Marked increases in border-zone wall thickness were also observed in SDF/GM-CSF-treated hearts (saline control, $1.41 \pm 0.16 \mathrm{~mm}$; GM-CSF only, $1.43 \pm 0.11 \mathrm{~mm}$; SDF only, $1.71 \pm 0.14 \mathrm{~mm}$; SDF/GM-CSF, $1.78 \pm 0.19 \mathrm{~mm}$ Figure 3, B). 
A.

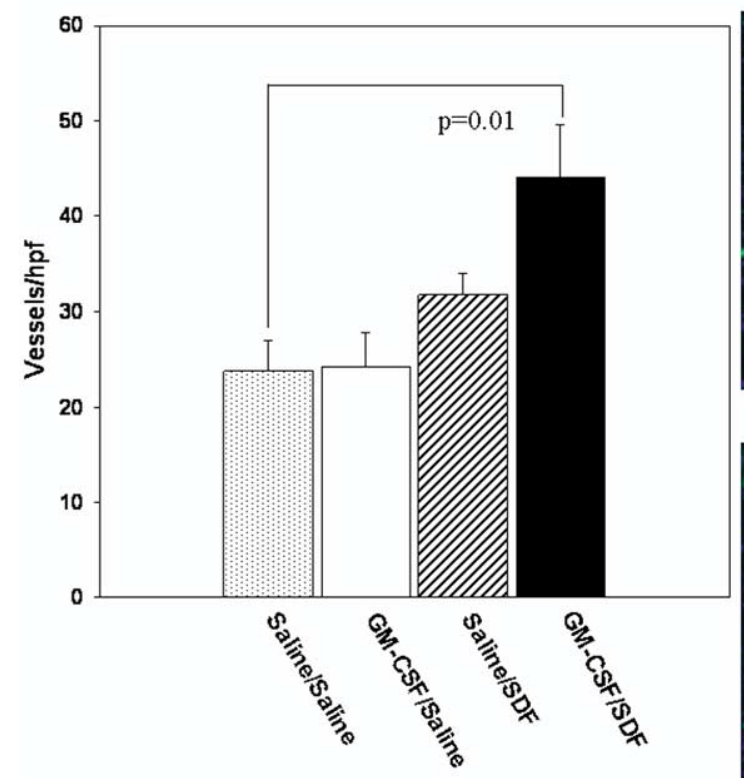

B.

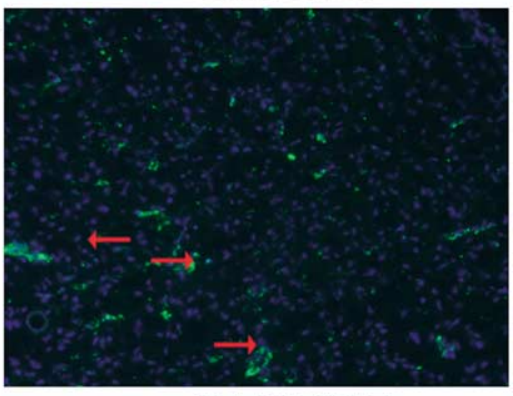

GM-CSF/SDF

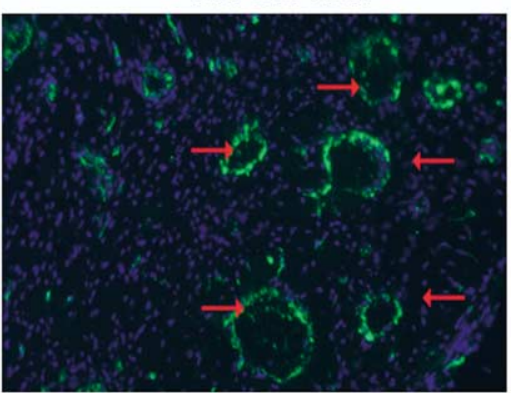

Figure 2. A, Graph of the mean number of vWF-positive blood vessels per high-powered field in each group. B, Representative images of myocardial sections depicting vWF (green)-expressing blood vessels for SDF/GM-CSFtreated and saline control animals. (Original magnification $40 \times$.) Arrows indicate vWF-positive vessels.

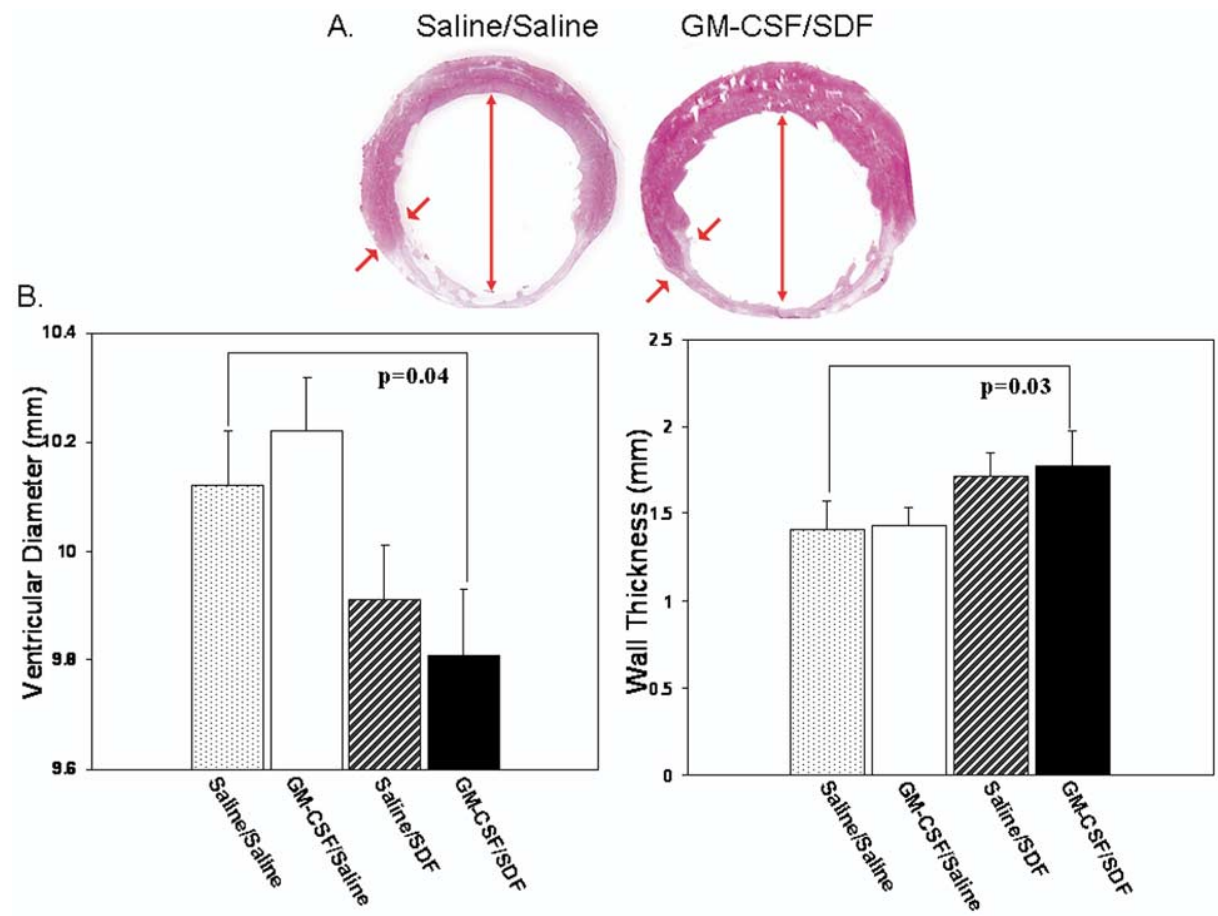

Figure 3. A, Representative hematoxylin and eosin-stained cross-sections of saline control and SDF/GM-CSFtreated hearts. Large arrows indicate where left ventricular diameter was measured, and small arrows indicate where border-zone thickness was measured. B, Graph of the mean ventricular diameter and wall thickness for each group. 
TABLE 1. Hemodynamic parameters of cardiac function 6 weeks after coronary ligation among control and SDF/GM-CSFtreated animals

\begin{tabular}{|c|c|c|c|c|c|}
\hline & $\begin{array}{c}A: \\
\text { Saline/saline } \\
(n=10)\end{array}$ & $\begin{array}{c}B: \\
\text { GMCSF/saline } \\
(n=10)\end{array}$ & $\begin{array}{c}C: \\
\text { Saline/SDF } \\
(n=10)\end{array}$ & $\begin{array}{c}D: \\
\text { GM-CSF/SDF } \\
(\mathrm{n}=10)\end{array}$ & $\begin{array}{c}\text { Groups significantly different } \\
\text { from } D\end{array}$ \\
\hline Heart rate (beats/min) & $185 \pm 10$ & $184 \pm 12$ & $205 \pm 8$ & $193 \pm 11$ & NS \\
\hline Maximum pressure $(\mathrm{mm} \mathrm{Hg})$ & $71.7 \pm 3.1$ & $70.6 \pm 1.7$ & $81.1 \pm 3.5$ & $93.9 \pm 3.2$ & $P<.001(A), .001(B), .05(C)$ \\
\hline Maximum $\mathrm{dP} / \mathrm{dt}(\mathrm{mm} \mathrm{Hg} / \mathrm{s})$ & $2602 \pm 201$ & $2756 \pm 101$ & $2886 \pm 120$ & $3515 \pm 303$ & $P<.01(A), .01(B), .05(C)$ \\
\hline Stroke volume $(\mu \mathrm{L})$ & $73.7 \pm 8.3$ & $80.6 \pm 24.6$ & $82.5 \pm 9.0$ & $117.8 \pm 16.8$ & $P<.01(A)$ \\
\hline Ejection fraction $(\%)$ & $28.1 \pm 3.1$ & $32.7 \pm 2.4$ & $40.4 \pm 2.2$ & $41.0 \pm 4.6$ & $P<.05(A), .05(B)$ \\
\hline Cardiac output (mL/min) & $13.3 \pm 1.3$ & $14.5 \pm 4.6$ & $15.7 \pm 1.2$ & $21.3 \pm 2.7$ & $P<.01(A), .05(B), .05(C)$ \\
\hline Stroke work $(\mathrm{mm} \mathrm{Hg} / \mu \mathrm{L})$ & $2780 \pm 781$ & $2921 \pm 1430$ & $3727 \pm 1736$ & $7185 \pm 973$ & $P<.001(A), .001(B), .01(C)$ \\
\hline Contractility slope $(\mathrm{mm} \mathrm{Hg} / \mu \mathrm{L})$ & $0.54 \pm 0.19$ & $0.58 \pm 0.15$ & $0.71 \pm 0.21$ & $1.7 \pm 0.4$ & $P<.01(A), .01(B), .05(C)$ \\
\hline
\end{tabular}

Measurements were obtained by using an intraventricular pressure-volume conductance catheter.

\section{Hemodynamics}

The SDF/GM-CSF-treated animals had statistically significant preservation of cardiac function compared with animals from the 3 other groups (Table 1). The SDF/GM-CSFtreated animals had significantly improved hemodynamics and systolic function, as measured on the basis of cardiac output, stroke volume, maximum left ventricular pressure, maximum $\mathrm{dP} / \mathrm{dt}$, and stroke work. SDF/GM-CSF-treated animals also had significantly improved cardiac contractility, as indicated by an increased slope of the pressurevolume relationship (saline control, $0.54 \pm 0.19 \mathrm{~mm} \mathrm{Hg}$ / $\mu \mathrm{L}$; GM-CSF only, $0.58 \pm 0.15 \mathrm{~mm} \mathrm{Hg} / \mu \mathrm{L}$; SDF only, $0.71 \pm 0.21 \mathrm{~mm} \mathrm{Hg} / \mu \mathrm{L} ; \mathrm{SDF} / \mathrm{GM}-\mathrm{CSF}, 1.7 \pm 0.4 \mathrm{~mm}$ $\mathrm{Hg} / \mu \mathrm{L})$. Representative pressure-volume loops from each group are shown in Figure 4.

\section{Discussion}

In an established model of ischemic cardiomyopathy, bone marrow stimulation with GM-CSF and targeted EPC chemokinesis with intramyocardial SDF enhanced myocardial neovascularization, attenuated adverse ventricular remodeling, and improved ventricular function. To our knowledge, this is the first report of this specific strategy of promoting targeted native endothelial cell revascularization for ischemic cardiomyopathy.

Beyond the standard medical and surgical therapies for ischemic cardiomyopathy, researchers have investigated many novel strategies addressing the underlying mechanisms of ventricular dysfunction. Ischemia, infarction, and subsequent loss of myocontractile mass form a clear mechanism of heart failure. However, chronic myocardial ischemia that does not yield tissue loss can induce a spectrum of derangements in cardiomyocyte metabolism, with marked decoupling of energy consumption and work production yielding an inefficient failing heart. This effect might be particularly pronounced in the relatively ischemic and dysfunctional border zone surrounding a myocardial infarct.
For example, in ischemic cardiomyopathy, high-energy creatinine phosphate stores are diminished, ${ }^{20}$ and in patients, decreased levels of myocardial creatine phosphate measured by phosphorous 31 magnetic resonance spectroscopy correlate highly with mortality. ${ }^{21}$ The activity of creatine kinase, an enzyme that catalyzes the high-energy phosphate transfer from creatine phosphate to adenosine diphosphate to form adenosine triphosphate, is also decreased. ${ }^{22}$ These factors initiate a deleterious cycle of myocardial contractile dysfunction and ventricular dilatation. Supraphysiologic stretch, cell-cell slippage, and geometric inefficiency with a progressive decrease in the ratio of wall thickness to chamber radius augment the tension load and promote further detrimental remodeling.

Enhancing myocardial perfusion might reverse metabolic derangements, restore energy efficiency, and reverse heart failure. Patients with postinfarction heart failure who have angiographic collateralization manifest improved regional ventricular function. ${ }^{23}$ Cardiomyopathic patients with anatomically amenable coronary disease also benefit from revascularization. ${ }^{2}$ Weeks to months after successful revascularization, patients will often exhibit increased ventricular ejection fraction on transthoracic echocardiography. ${ }^{24}$ Unfortunately, the majority of patients with heart failure do not have coronary disease anatomically correctable by percutaneous or operative techniques, and transmyocardial laser revascularization has had mixed results. Molecular and cellular revascularization strategies are under active investigation and have included the use of angiogenic factors that appear to act, at least in part, through the induction of EPC-mediated neovasculogenesis. Although angiogenesis is generally defined as the sprouting of new vessels from existing vessels through the proliferation of mature endothelial cells and vasculogenesis refers to the embryologic formation of blood vessels from EPCs, these terms are often used interchangeably in the literature. EPC- 

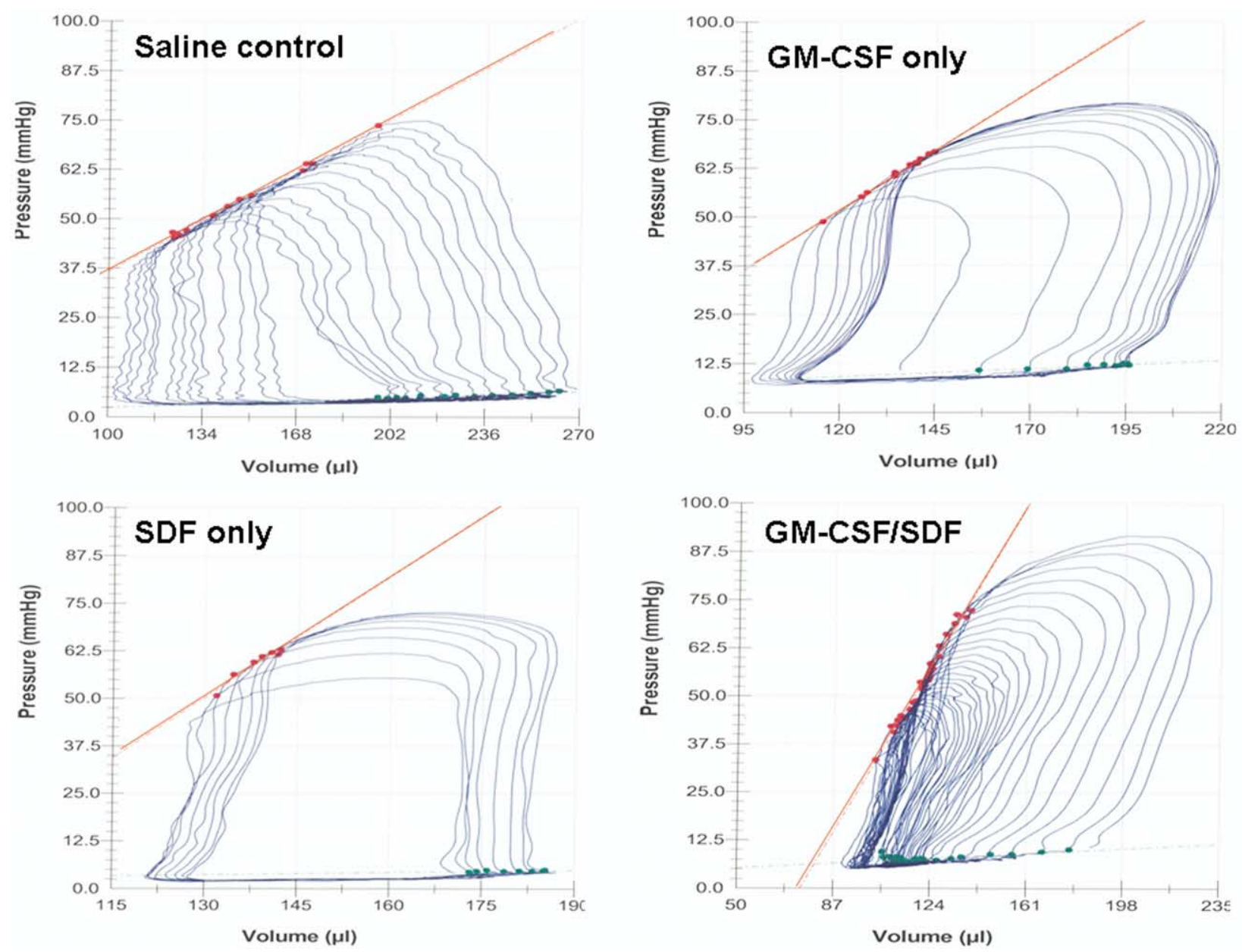

Figure 4. Improved contractility was seen among SDF/GM-CSF-treated animals (slope, $1.7 \pm 0.4 ; x$-intercept, 76.2 $\pm 21.3 \mu \mathrm{L} ; \mathrm{r}=0.96 ; n=10$ ) when compared with control animals (slope, $0.54 \pm 0.19$; $x$-intercept, $122.1 \pm 15.2 \mu \mathrm{L}$; $r=0.78 ; n=10 ; P<.01)$. Representative pressure-volume loops for all 4 groups are displayed.

mediated blood vessel development in adults is generally believed to occur through a combination of angiogenic and vasculogenic processes. ${ }^{25}$

Combining the sequentially complementary approaches of global bone marrow stimulation and targeted EPC chemokinesis, we devised a strategy of endogenous myocardial repair for ischemic cardiomyopathy. The observed lack of efficacy of GM-CSF therapy alone possibly could be due to the fact that multiple tissues express native SDF, and thus bone marrow stimulation with only GM-CSF is unlikely to yield sufficient myocardium-specific targeting of EPCs. In a rabbit hind-limb model of ischemia, repeated daily dosing of GM-CSF for 7 days before femoral artery ligation yielded increased vascularity. ${ }^{11}$ This treatment approach is limited in its extent of clinical translatability. The observed lack of efficacy of lone intramyocardial SDF therapy is probably due to limitations of insufficient bone marrow production of EPCs. In fact, the injection of only SDF into ischemic hind limbs has been found to result in autoamputation. ${ }^{17}$

In contrast to most research groups, which administer therapeutic agents at the time of coronary ligation, we used a model of ischemic cardiomyopathy in which the animals first underwent infarction, followed by 3 weeks of remodeling. By waiting 3 weeks after infarction, the animals had sufficient time to experience some degree of heart failure, thus allowing for a more clinically relevant analysis. Also, this model permitted the elimination of potential infarct modulation from confounding the results. At the time of reoperation, animals were first randomized to the different groups to minimize any potential minor differences in animal size, infarct size, or degree of remodeling. The validity of the proximal LAD ligation model has been confirmed by multiple research groups. In our own studies of this model, 
rats manifested anterolateral infarcts that reproducibly comprised $30 \%$ of the left ventricle, which is consistent with results previously reported. ${ }^{18}$ The observation of equivalent infarct sizes in all 4 groups in this study precludes attributing any hemodynamic differences to disparities in infarct size. The stability in infarct size over this period of time is also consistent with previously reported results. ${ }^{18}$ Furthermore, with regard to localization of intended therapy, in the current study SDF was directly injected into the border zone, where neovascularization would potentially improve contractility, as opposed to the scar, where benefit was less likely to be expected.

An immunofluorescent von Willebrand assay used to label endothelial cells lining border-zone blood vessels was useful in determining the vasculogenic states of experimental groups. As indicated by increased vessel densities in SDF/GM-CSF-treated border zones, we have detected improvements in myocardial vascularity relative to hearts treated with saline. These findings warrant further study of regional perfusion estimations through functional vascularization assessment with techniques such as radionuclide imaging or tomato lectin histologic angiography. ${ }^{26} \mathrm{We}$ have previously reported in a related model that increased vascularity, as evidenced by von Willebrand staining, correlated with increased microsphere perfusion. ${ }^{5}$

Overall, the observed finding of a marked increase in cells coexpressing CD34 and VEGFR2 in SDF/GM-CSFtreated hearts compared with levels in the saline control group warrant extensive future studies in tracking the mobilization and differentiation of EPCs in this experimental model. Bone marrow and peripheral blood flow cytometry and further myocardial immunohistochemical analysis of EPC markers in native, control, and SDF/GM-CSF-treated animals immediately after treatment and at various time points could better delineate the proliferation, mobilization, targeting, and differentiation of the EPCs.

The effect of SDF/GM-CSF treatment on ventricular remodeling was analyzed by using histologic sections. The increased border-zone wall thickness and decreased chamber dilatation imply an alteration in the detrimental remodeling process with SDF/GM-CSF therapy. These results must be viewed within the context of the limitations of measurement precision for submillimeter changes. Interestingly, a pilot microscopic study of border-zone cardiomyocyte numbers revealed more cells in the SDF/GM-CSF group than in the saline group $(40.0 \pm 5.6$ vs $27.0 \pm 6.0$ cells/hpf, $\mathrm{n}=5, P=.011$ ), findings consistent with the observed increased border-zone wall thickness and potentially caused by increased regional vascularity and tissue preservation. As an internal control, we observed no difference in the number of cardiomyocytes per high-power field in remote myocardium between SDF/GM-CSF-treated and saline-only hearts $(63.2 \pm 3.0$ vs $63.0 \pm 3.2$ cells $/ \mathrm{hpf}, P=$
NS). For more detailed studies into the time course progression of ventricular remodeling, serial echocardiography or magnetic resonance imaging could be used. These imaging modalities could potentially also provide regional contractility data.

The improved cardiac function observed in the SDF/ GM-CSF group is significant when compared with that seen in the other 3 groups. When comparing these animals with native noninfarcted animals, the cardiac function appears to be close to normal. For example, in a separate group of ageand weight-matched native Lewis rats $(\mathrm{n}=5)$ studied for hemodynamics, heart rate was $194 \pm 15$ beats/min, maximum pressure was $103 \pm 12.4 \mathrm{~mm} \mathrm{Hg}$, maximum $\mathrm{dP} / \mathrm{dt}$ was $4582 \pm 346 \mathrm{~mm} \mathrm{Hg} / \mathrm{s}$, ejection fraction was $39.4 \% \pm 4.9 \%$, cardiac output was $25.7 \pm 5.5 \mathrm{~mL} / \mathrm{min}$, and contractility slope was $1.8 \pm 0.3 \mathrm{~mm} \mathrm{Hg} / \mu \mathrm{L}$.

Although not directly investigated in this study, the improved hemodynamics might be the result of a combined effect of somewhat improved ventricular geometry and increased border-zone contractility from restored vascularization. The ability to demonstrate reversal of regional borderzone cardiomyocyte ischemia in future studies would strongly support this hypothesis. A possible alternative explanation for many of the observed findings would be the stimulation of cardiac progenitor cells. This seems less likely with data illustrating increases in endothelial cellspecific markers. There also appears to be an increasing body of evidence to suggest that bone marrow-derived progenitor cells differentiate primarily into endothelial cells and only rarely into cardiomyocytes. ${ }^{27,28}$ In a unique clinical model of myocardial repair, female donor hearts (XX) transplanted into male recipients (XY), when examined later for Y-chromosome positive cells, have demonstrated predominantly endothelial lineage cells and very few cardiomyocytes. $^{29}$

The EPC proliferation and targeting strategy described in this report could potentially be applied to a variety of ischemic disease processes. One could also envision SDFcoated intravascular grafts, stents, prosthetic valves and rings, patches, anastomotic devices, and ventricular assist devices to promote endothelialization.

In summary, this study describes GM-CSF mobilization and intramyocardial SDF chemokinesis of EPCs to form a unique neovascularization treatment strategy for ischemic cardiomyopathy and demonstrates enhanced vascularity, attenuated adverse ventricular remodeling, and improved myocardial function.

\section{References}

1. Braunwald E, Bristow MR. Congestive heart failure: fifty years of progress. Circulation. 2000;102:14-23.

2. Elefteriades JA, Tolis G Jr, Levi E, Mills LK, Zaret BL. Coronary artery bypass grafting in severe left ventricular dysfunction: excellent 
survival with improved ejection fraction and functional state. $J \mathrm{Am}$ Coll Cardiol. 1993;22:1411-7.

3. Sellke FW, Ruel M. Vascular growth factors and angiogenesis in cardiac surgery. Ann Thorac Surg. 2003;75(suppl):S685-90.

4. Rosengart TK, Lee LY, Patel SR, Sanborn TA, Parikh M, Bergman GW, et al. Angiogenesis gene therapy: pha7se I assessment of direct intramyocardial administration of an adenovirus expressing VEGF121 cDNA to individuals with clinically significant severe coronary artery disease. Circulation. 1999;100:468-74.

5. Jayasankar V, Bish LT, Pirolli TJ, Berry MF, Burdick J, Woo YJ. Gene transfer of hepatocyte growth factor attenuates postinfarction heart failure. Circulation. 2003;108(suppl II):II230-6.

6. Kocher AA, Schuster MD, Szabolcs MJ, Takuma S, Burkhoff D, Wang $\mathrm{J}$, et al. Neovascularization of ischemic myocardium by human bone-marrow-derived angioblasts prevents cardiomyocyte apoptosis, reduces remodeling and improves cardiac function. Nat Med. 2001;7: 430-6.

7. Yin AH, Miraglia S, Zanjani ED, Almeida-Porada G, Ogawa M, Leary AG, et al. AC133, a novel marker for human hematopoietic stem and progenitor cells. Blood. 1997;90:5002-12.

8. Walter DH, Rittig K, Bahlmann FH, Kirchmair R, Silver M, Murayama $T$, et al. Statin therapy accelerates reendothelialization: a novel effect involving mobilization and incorporation of bone marrow derived endothelial progenitor cells. Circulation. 2002;105:3017-24.

9. Peichev M, Naiyer AJ, Pereira D, Zhu Z, Lane WJ, Williams M, et al. Expression of VEGFR-s and AC133 by circulating human CD34+ cells identifies a population of functional endothelial precursors. Blood. 2000;95:952-8.

10. Jackson KA, Majka SM, Wang H, Pocius J, Hartley CJ, Majesky MW, et al. Regeneration of ischemic cardiac muscle and vascular endothelium by adult stem cells. J Clin Invest. 2001;107:1395-402.

11. Takahashi T, Kalka C, Madusa H, Chen D, Silver M, Kearney M, et al. Ischemia and cytokine-induced mobilization of bone marrow-derived endothelial progenitor cells for neovascularization. Nat Med. 1999;5:434-8.

12. Mohle R, Bautz F, Rafii S, Moore MA, Brugger W, Kanz L. The chemokine receptor CXCR-4 is expressed on CD34+ hematopoietic progenitors and leukemic cells and mediates transendothelial migration induced by stromal cell-derived factor-1. Blood. 1998;91:4523-30.

13. Kim $\mathrm{CH}$, Broxmeyer HE. In vitro behavior of hematopoietic progenitor cells under the influence of chemoattractants: stromal cell-derived factor-1, steel factor, and the bone marrow environment. Blood. 1998; 91:100-10.

14. Aiuti A, Webb IJ, Bleul C, Springer T, Guitierrez-Ramos JC. The chemokine SDF-1 is a chemoattractant for a human CD34+ hematopoietic progenitor cells and provides a new mechanism to explain the mobilization of CD34+ progenitors to peripheral blood. J Exp Med. 1997;185:111-20.

15. Mirshahi F, Pourtau J, Li H, Muraine M, Trochan V, Legrand E, et al. SDF-1 activity on microvascular endothelial cells: consequences on angiogenesis in in vitro and in vivo models. Thromb Res. 2000;99:587-94

16. Pillarisetti K, Gupta SK. Cloning and relative expression analysis of rat stromal cell derived factor-1 (SDF-1): SDF-1 alpha mRNA is selectively induced in rat model of myocardial infarction. Inflammation. 2001;25:293-300.

17. Yamaguchi J, Kusano KF, Masuo O, Kawamoto A, Silver M, Murasawa $\mathrm{S}$, et al. Stromal cell-derived factor-1 effects on ex vivo expanded endothelial progenitor cell recruitment for ischemic neovascularization. Circulation. 2003;107:1322-8.

18. Liu YH, Yang XP, Nass O, Sabbah HB, Peterson E, Carretero OA. Chronic heart failure induced by coronary artery ligation in Lewis inbred rats. Am J Physiol. 1997;272:H722-7.

19. Woo YJ, Taylor MD, Cohen JE, Jayasankar V, Bish LT, Burdick J, et al. Ethyl pyruvate preserves cardiac function and attenuates oxidative injury after prolonged myocardial ischemia. $J$ Thorac Cardiovasc Surg. 2004;127:1262-9.

20. Ingwall JS. Is cardiac failure a consequence of decreased energy reserve? Circulation. 1993;87(suppl VII):VII58-62.

21. Neubauer S, Horn M, Cramer M, Harre K, Newell JB, Peters W, et al. Myocardial phosphocreatine-to-ATP ratio is a predictor of mortality in patients with dilated cardiomyopathy. Circulation. 1997;96:2190-6.

22. Nascimben I, Ingwall JS, Pauletto P, Friedrich J, Gwathmey JK, Saks $\mathrm{V}$, et al. Creatine kinase system in failing and nonfailing human myocardium. Circulation. 1996;94:1894-901.

23. Ejiri M, Fujita M, Sakai O, Miwa K, Asanoi H, Sasayama S. Development of collateral circulation after acute myocardial infarction; its role in preserving left ventricular function. J Cardiol. 1990;20:31-7.

24. Tolis GA Jr, Korkolis DP, Kopf GS, Elefteriades JA. Revascularization alone (without mitral valve repair) suffices in patients with advanced ischemic cardiomyopathy and mild-to-moderate mitral regurgitation. Ann Thorac Surg. 2002;74:1476-81.

25. Szmitko PE, Fedak PWM, Weisel RD, Stewart DJ, Kutryk MJB, Verma S. Endothelial progenitor cells: new hope for a broken heart. Circulation. 2003;107:3093-100.

26. Gee MS, Saunders HM, Lee JC, Sanzo JF, Jenkins WT, Evans SM, et al. Doppler ultrasound imaging detects changes in tumor perfusion during antivascular therapy associated with vascular anatomic alterations. Cancer Res. 2001;61:2974-82.

27. Murray CE, Soonpa MH, Reinecke H, Hidehiro N, Nakajima HO, Rubart M, et al. Hematopoietic stem cells do not transdifferentiate into cardiac myocytes in myocardial infarcts. Nature. 2004;428:664-8.

28. Nygren JM, Jovinge S, Breitbach M, Sawen P, Roll W, Hescheler J, et al. Bone marrow-derived hematopoietic cells generate cardiomyocytes at a low frequency through cell fusion but not transdifferentiation. Nat Med. 2004;10:494-501.

29. Quaini F, Urbanek K, Beltrami AP, Finato N, Beltrami CA, NadalGinard B, et al. Chimerism of the transplanted heart. $N$ Engl J Med. 2002;346:5-15. 